\title{
Duroplasty in iatrogenic dorsal spinal cord herniation: illustrative case
}

\author{
Ikenna Ogbu, MBBS, MPH, Mohamed Eltoukhy, MD, PhD, and Nikolaos Tzerakis, MBBS \\ Department of Neurosurgery, Royal Stoke University Hospital, University Hospitals of North Midlands (UHNM), Staffordshire, West Midlands, United Kingdom
}

BACKGROUND The case report detailed an unusual presentation of an iatrogenic dorsal cord herniation at the level of the thoracic cord after insertion of an epidural catheter 8 months before presentation to the neurosurgical clinic.

OBSERVATIONS Only 13 cases of iatrogenic dorsal cord herniation, most of which occurred after spinal surgery, have been described in the literature. This was the first case of a spinal cord hernia described after the insertion of an epidural catheter. In this case study, the authors described a 38-year-old man who presented with progressive lower limb weakness, sensory deficits, perianal numbness, and urinary/fecal incontinence. He was diagnosed with a spinal cord hernia that reherniated after an initial sandwich duroplasty repair. Definitive repair was made after his re-presentation using an expansile duroplasty.

LESSONS In patients with previous spinal instrumentation who present with neurological symptoms, spinal cord herniation should be considered a likely differential despite its rarity. In this case, a simple duroplasty was insufficient to provide full resolution of symptoms and was associated with recurrence. Perhaps a combination of graft and expansile duroplasty may be used for repair, especially when associated with a tethered cord and in the presence of significant adhesions.

https://thejns.org/doi/abs/10.3171/CASE21347

KEYWORDS spinal cord herniation; spinal duroplasty; spinal cord hernia repair

Spinal cord herniation in and of itself has been noted to be a rare phenomenon and should be suspected in individuals who present with an unusual combination of motor/sensory and autonomic deficits. ${ }^{1}$ Dorsal herniation of the cord due to iatrogenic causes is an even more uncommon phenomenon, with only 13 reported cases in the literature to date (Table 1), and these cases have been most commonly due to spinal surgery.

Its association with previous epidural analgesia is even less commonly encountered, and in this case report, we describe the presentation and management of dorsal cord herniation in a patient with complications that may have been secondary to an epidural catheter insertion after a bilateral sequential single lung transplant 5 months before initial presentation.

Posterior cord herniation has been described after trauma, but iatrogenic and idiopathic (due to congenital dural weakness) cases are reported to occur more commonly. ${ }^{2}$ This case report is the first description of an iatrogenic herniation occurring after insertion of an epidural catheter.

\section{Illustrative Case}

A 38-year-old man presented with a posterior cord herniation at T4 with no history of previous trauma. The history started 5 months before admission to our unit, when he was admitted for a bilateral lung transplant as treatment for his refractory cystic fibrosis. That surgery was mostly uneventful. However, to better manage his pain, an epidural catheter was inserted, the first attempt of which failed.

Eight months after his lung transplant, he presented with reduced sensations in both lower limbs and reports of both urinary and fecal incontinence. He was then referred to the neurosurgical clinic where he was assessed. He described a progressive loss of sensation that started in his right ankle and spread to involve the whole of the right lower limb. Additionally, he reported repeated episodes of both urinary and fecal incontinence. On examination, he had a sensory level at T6, with decreased sensation below his scar on the right side of his trunk. There was also associated dysaesthesia and paraesthesia and loss of temperature on the right side as well as perianal numbness. He was 


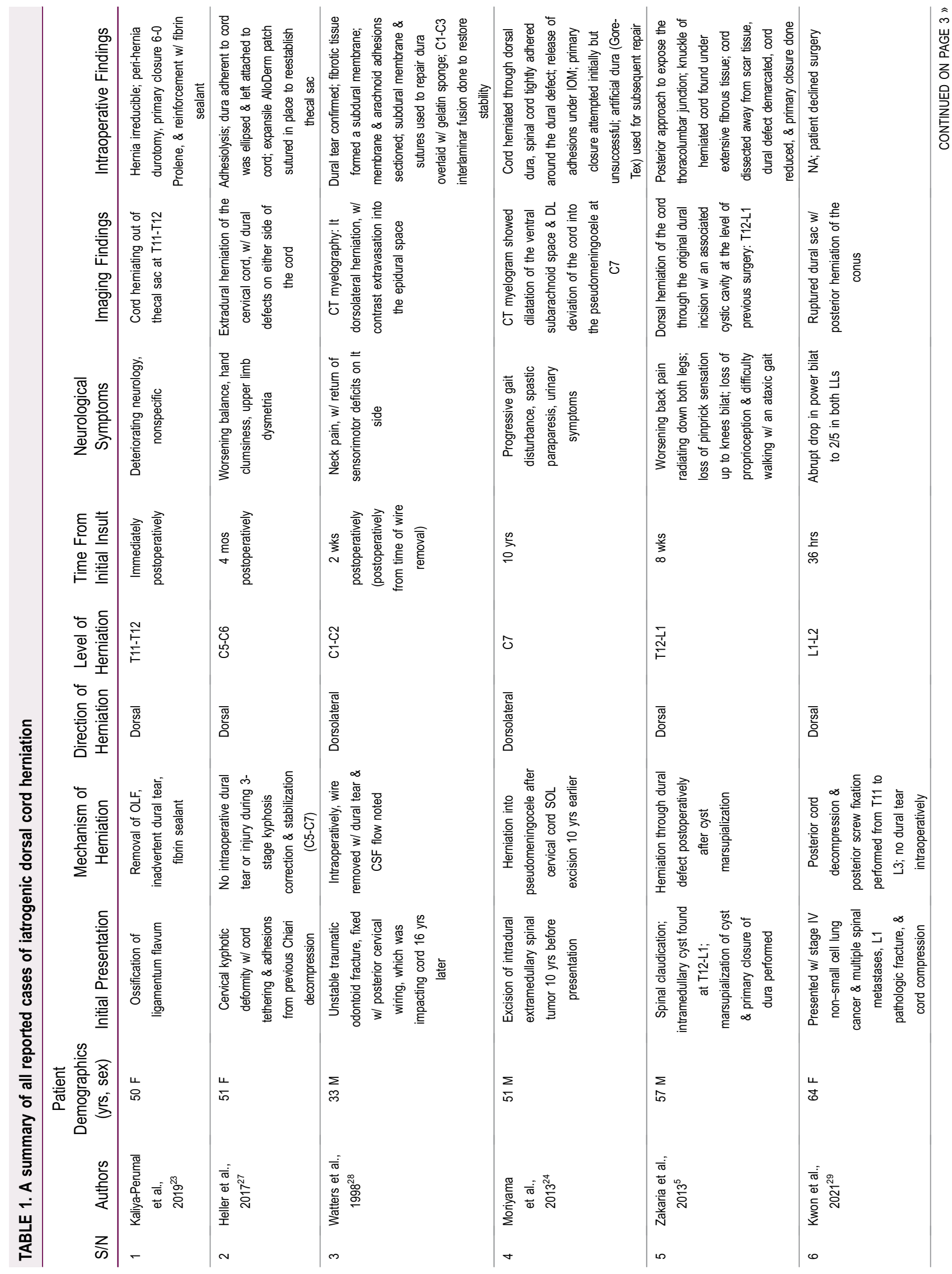




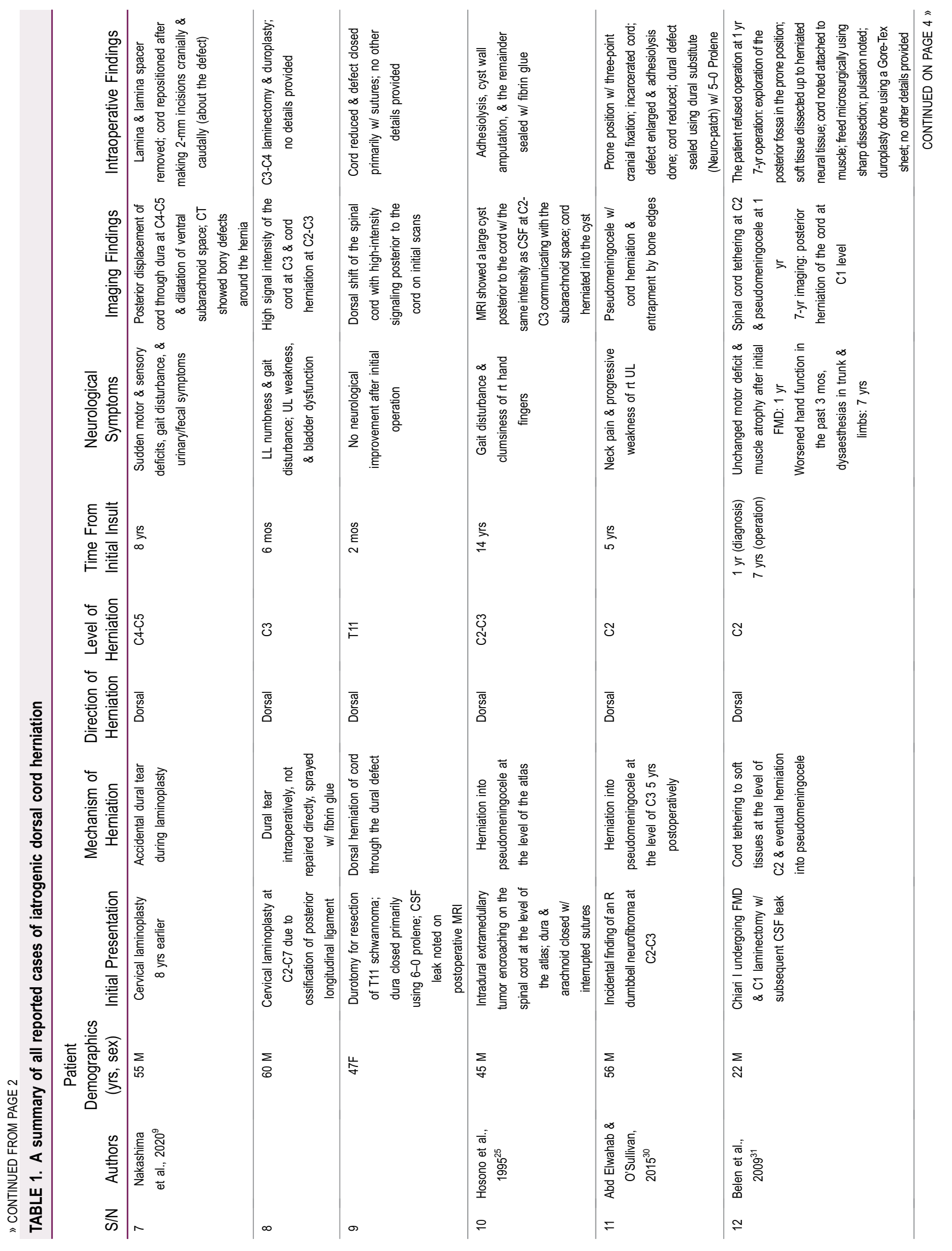




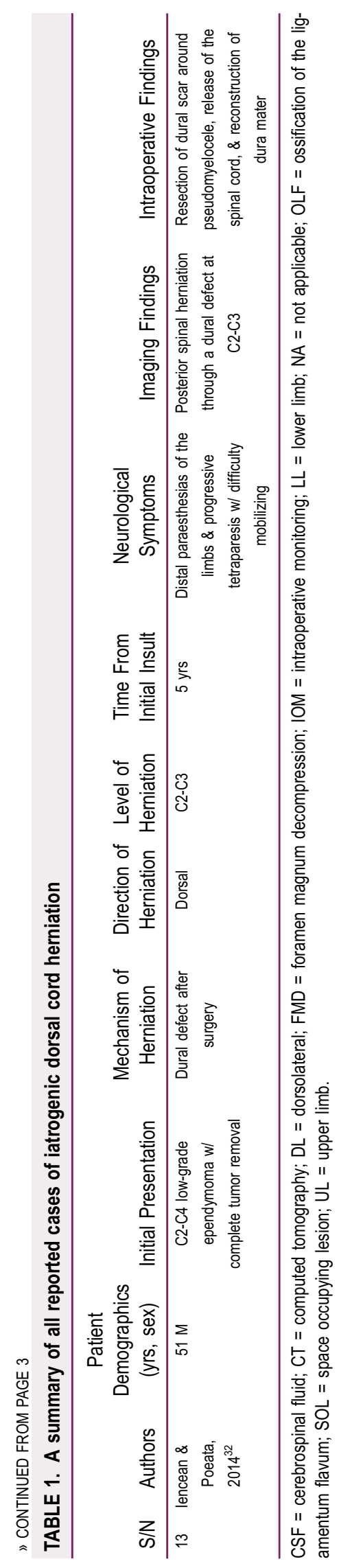

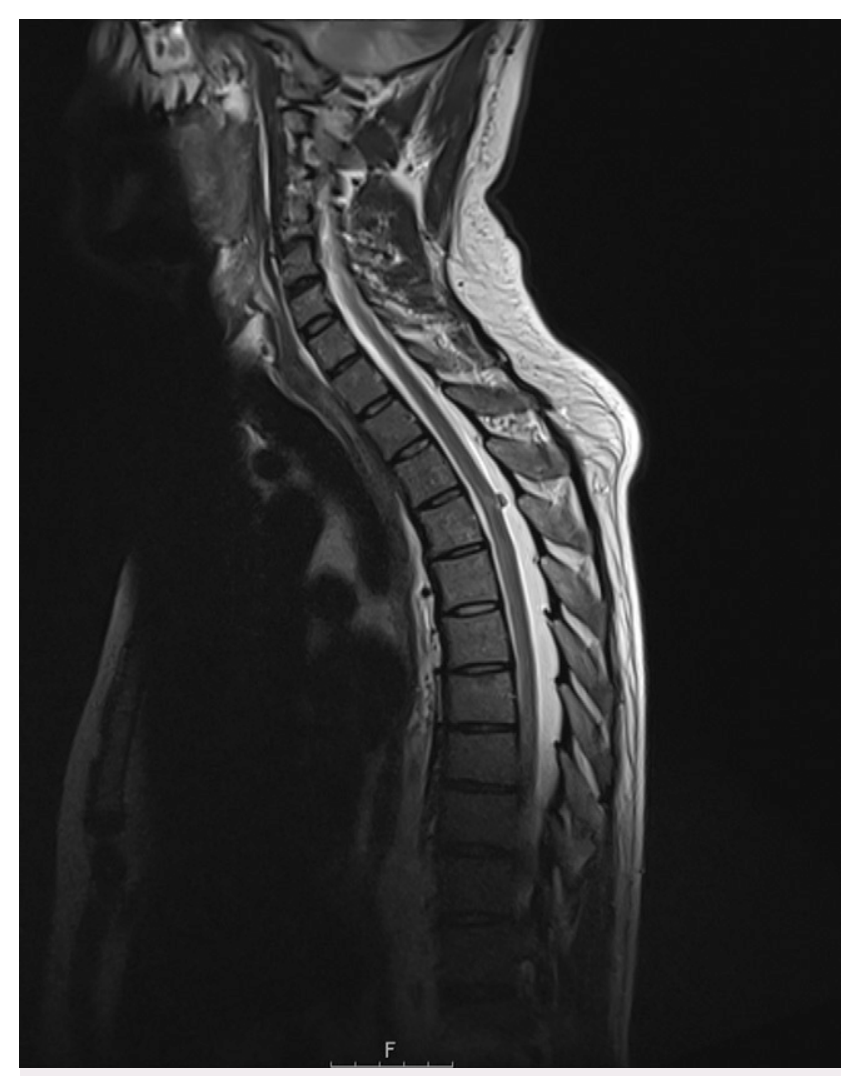

FIG. 1. T1 MRI sequence showing the initial dorsal protrusion of the dorsal cord preoperatively.

noted to have bilaterally brisk tendon reflexes, with clonus in both ankles and bilateral flexor plantar response. However, he had intact motor power in all muscle groups. There was no history of mechanical trauma or previous spinal operations.

A magnetic resonance imaging (MRI) scan (Fig. 1) showed a dorsal cord herniation and posterior breaking of the cord with T2 high-signal intensity of the central and dorsal cord at the level of T4.

Considering the history, presenting signs, and postpresentation MRI scan, the suspicion was that of a spinal cord hernia at the T4 level. The patient was thus admitted a week later for surgical repair of the cord herniation. During the initial operation, the herniated cord was noted to have "mushroomed" through a small $10-\mathrm{mm}$ dorsal dural defect with adhesions to the dura on the outside. Dural and arachnoid adhesions formed a ring around the herniated cord and incarcerated it (Fig. 2). Dissection was started by making two dural midline snip incisions cranial and caudal to the herniated cord. From the cranial opening, careful dissection was performed in the caudad direction until encountering the incarcerating ring and vice versa from the caudal opening. Careful motor evoked potentials-guided adhesiolysis was performed around the incarcerated cord to separate it from the dura. The herniated cord was completely released but never restored to a complete normal morphology, with persistent bleb-like swelling at the location of the herniation. Further dissection would potentially endanger the neural tissue, and eventually, the cord was untethered and returned to its normal position within the thecal sac (Fig. 3), where it was pulsating normally. The dural opening was approximately $25 \mathrm{~mm}$ at the end in craniocaudal 


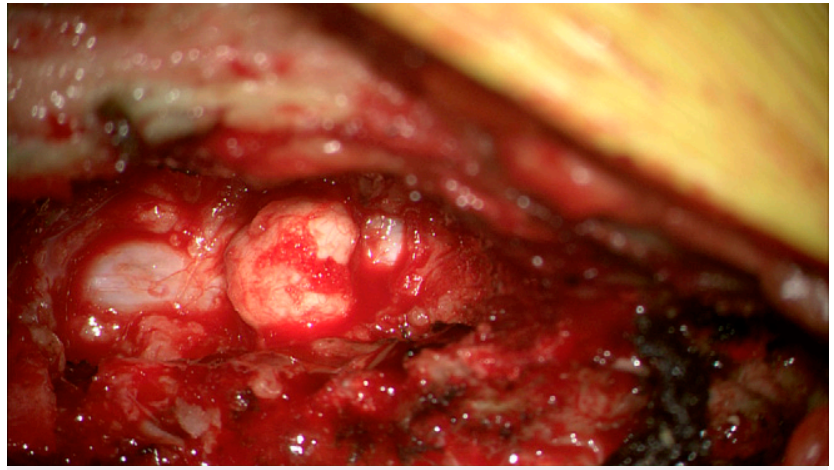

FIG. 2. After access into the skin, obvious incarceration of the cord by dural and arachnoid adhesions is visible, forming a ring around the herniated cord.

diameter, with the reduced cord seen freely pulsating inside. Attempts at primary repair would have ended in compression on the cord, so the dura was left open. In situ synthetic dural substitutes were used as onlay (above) and inlay (below) for this opening (also called the sandwich technique). ${ }^{3,4}$ Stitches without tension were used to secure the grafts to avoid misplacement.

The patient made a good clinical recovery with improved sensations in both lower limbs. He was discharged home a few days later. Unfortunately, 2 weeks after the operation, he presented again with a 48-hour history of sensory deterioration in both lower limbs. Deep sensations were particularly affected. The picture was suggestive of posterior cord syndrome.

Reexploration was decided. Intraoperative findings were as follows: the cord was herniating through the surgical dural opening and pushing the onlay grafts dorsally. A false incarceration ring was created by the edges of the dural opening. It was clear that the cord had retethered although the inlay graft was still in place.

Onlay dural grafts were removed. The dural opening was extended approximately 5 to $10 \mathrm{~mm}$ in both cranial and caudal directions. A sleeve of fascia with fat was inserted and sutured in place to the dural edges. This time, dural hitch stitches were applied between the dura and the paraspinal muscles to create a tent-like space behind the cord. Adequate expansile duroplasty was achieved at the end with ample artificial dorsal subdural space. Synthetic dural sealants were used, with no cerebrospinal fluid effluence noted at the end.

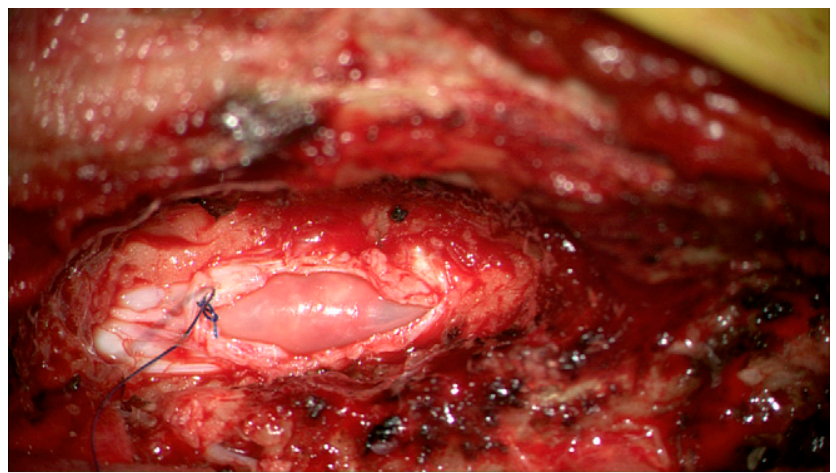

FIG. 3. Cord reduced into intrathecal sac before closure using an expansile duroplasty.

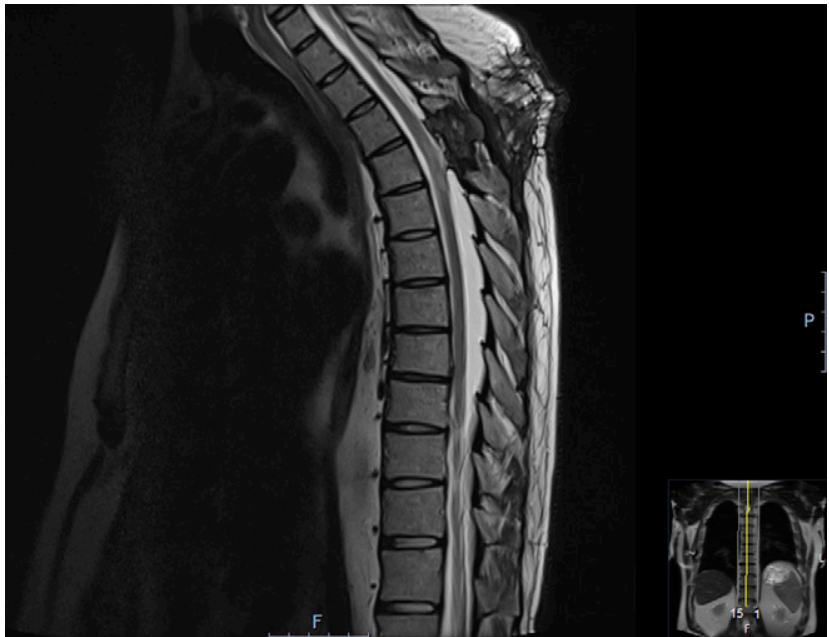

FIG. 4. A T2 MRI short T1 inversion recovery sequence showing improved herniation of the cord at clinic follow-up. The inset picture shows the plane at which the larger sagittal slice (the bigger picture) was taken.

After the operation, the patient made excellent progress neurologically as well as with the physiotherapy team and was discharged 18 days after the second operation. He subsequently has made a good recovery. A delayed postoperative MRI scan at follow-up (Fig. 4) shows improved cord edema. Clinically, the patient self-reports a slow improvement in proprioception as well as a return of bladder sensation and a reduction in the frequency of the episodes of urinary incontinence and no new reported episodes of fecal incontinence.

\section{Discussion}

Spinal cord herniation is described in the literature as a rare cause of spinal cord dysfunction, ${ }^{1,5-8}$ which is often amenable to surgery. It can commonly be classified into spontaneous, iatrogenic, and posttraumatic. Spontaneous and idiopathic presentations have been described more commonly in the literature, and herniation due to a traumatically acquired dural defect is the least reported cause. ${ }^{1}$ latrogenic dorsal cord herniation has been described even more rarely, ${ }^{9}$ with only 13 cases reported in the literature to date (Table 1).

Generally, most herniations have been described in the ventral and/or ventrolateral spinal cord, most commonly at the T4-T5 level. ${ }^{8}$ Spontaneous dorsal cord herniation has been described even more rarely, with one case described in a 2-year-old boy secondary to herniation into an extradural arachnoid cyst. ${ }^{10}$

The presentation of spinal cord herniation is reportedly variable, but more than $50 \%$ of cases present with Brown-Sequard syndrome, which is suggestive of cord hemisection. ${ }^{11}$ Commonly, they also present with spastic paraparesis, a dysfunctional bladder/dysfunctional bowel (up to $71 \%$ of patients), lower limb pain, and sometimes low-pressure headaches. ${ }^{8}$ When associated with tethering, the presentation may include pain (in the lower back or lower extremities), lower limb weakness, and sensorimotor dysfunction unrelated to dermatomal distribution.

The pathophysiological processes for developing spinal cord herniations are not well understood. However, as much as the entities of tethered cord and tethered cord syndrome have been described differently, with the latter described as a tethering of the filum, their pathophysiological mechanisms share some similarity. ${ }^{12-16}$ In 
in vivo models, the pathophysiological mechanism of spinal cord malfunction in cord tethering was postulated to be due to metabolic dysfunction in stretched and incarcerated neurons, which results in ischemia because of anterior spinal artery involvement and increased cord susceptibility to hypoxic injury due to distortion of the smaller vessels around the cord. ${ }^{16,17}$ Also, traction-induced ischemia has been linked to reduced conduction, leading to both reversible and irreversible nerve damage. ${ }^{12-15,18}$ Clinical progression in cord tethering has been postulated to be stretch-related damage to the herniated/tethered fibers in the lateral funiculus involving the spinothalamic and, subsequently, descending tracts. ${ }^{8,13}$ The vascular mechanism has also been postulated to explain the lack of improvement in some patients after untethering. ${ }^{16}$

Making a diagnosis of spinal cord herniation is difficult because this entity is classically rare and not the foremost diagnosis on most neurologists'/neurosurgeons' minds, with the median time to diagnosis approximately 17 months and one patient having a 16-year time to diagnosis. ${ }^{19}$ This delay in diagnosis typically leads to unnecessary testing, visits to the doctor, imaging, and procedures that may have no benefit to the patient. Tethered cord in itself is notoriously difficult to diagnose, with most cases presenting with a long history of misdiagnosis before being correctly diagnosed. ${ }^{16}$

There seem to be two general types of herniated cord according to the intraoperative morphology. The first type occurs in small defects $(<1-2 \mathrm{~cm})$ within a relatively short time of insult. ${ }^{20}$ In this type, adhesions are minimal, and herniation is mainly driven by the differential pressure between the subdural and extradural spaces. Good primary closure seems to be sufficient. The second type occurs in larger defects $(>1-2 \mathrm{~cm})$ with longer time intervals. Adhesions are the main pathology, and primary repair is difficult and almost impossible. ${ }^{20}$ The current mainstay of treatment of this type involves careful microdissection to release all surrounding adhesions, returning the cord to its original position, and effecting closure using one of two techniques: the enlargement of the inner dural defect, first described by Watanabe et al., ${ }^{21}$ which was our initial approach during the first operation, or a dural graft as propounded by Zairi et al., ${ }^{22}$ which was our second approach. ${ }^{21,22}$

We reviewed the literature that reported similar cases with an iatrogenic dorsal cord herniation (Table 1). Special consideration was given to the surgical technique used and its correlation with the presentation.

Primary closure was attempted successfully in three cases. ${ }^{5,9,23}$ Those cases had the following factors in common: a short interval between the time of insult and time of presentation (ranging between immediately postoperative to 8 weeks), being in the thoracic region, and having smaller dural defects.

On the other hand, primary closure was performed unsuccessfully in one case reported by Moriyama et al. in 2013. ${ }^{24}$ That case had a 10-year interval between the primary cervical operation and the new presentation. The dural defect was larger, with thick surrounding dural adhesions and cord tethering. A different strategy was retried successfully that involved subsequent secondary repair with artificial dura using an expansile duroplasty technique.

Another technique that was used by Nakashima et al. and Hosono et al. involved careful adhesiolysis, amputation of any pseudocysts, reduction of the cord to the thecal sac, and enlargement of the dural opening in both cranial and caudal directions. ${ }^{9,25}$ This is done in combination with or without an inlay graft and a dural sealant.

In cases with long-standing pathology with larger defects when thick adhesions are the main driving force of tethering, varying degrees of bony decompression were done at first to gain safe access to the herniated cord. This was followed by adhesiolysis and dural opening enlargement, as described in the former technique. There is an added use of grafts, either synthetic or autologous. The graft is sutured in place to create an adequate space behind the cord and create expansive duroplasty.

After surgical intervention, a significant improvement in symptoms has been reported, with $78 \%$ (with back pain at presentation) and $83 \%$ (with leg pain at presentation) of patients reporting improved symptoms. ${ }^{26}$

\section{Observations}

For this index patient who presented with an insidious onset of symptoms predominantly suggestive of a posterior cord syndrome, we hypothesize that the precipitating event was the insertion of the epidural catheter after his bilateral lung transplant.

Dorsal cord herniation associated with tethering has rarely been reported in the literature, and unsurprisingly in our index case, it was misdiagnosed repeatedly in the initial workup period, which is commonly encountered with tethered cord. ${ }^{26}$

To remedy his symptoms, which had gotten worse over time, we decided on a surgical approach. Our initial approach was adhesiolysis with enlarging of dural opening and inlay graft, which failed and resulted in retethering. Reoperation was needed with a more extensive expansion duroplasty and closure with an inlay graft with a tenting technique of the dura. That approach was successfully done to reduce the possibility of retethering.

\section{Lessons}

Dorsal cord herniation (especially iatrogenic) has been rarely reported in the literature, even less frequently in association with cord tethering. Spinal cord herniation is a rare phenomenon that occurs more commonly as either a spontaneous or idiopathic herniation. The diagnosis of cord herniation possibly with associated tethering should be considered in patients with a bizarre combination of upper motor neuron neurology that cannot be attributed to any other central neurological lesion. Additionally, because of the proposed pathophysiological mechanism of vascular injury in cord tethering, there is a chance of no improvement after detethering of the cord in some patients.

Also, during the management of this case, we attempted a singular technique for closure using graft duroplasty, which resulted in retethering. Therefore, it may be worth considering a combination of both expansion and graft duroplasty for the management of dorsal cord herniation, more so in association with tethering in the presence of significant adhesions. However, more research is required to determine if this is associated with a decreased rate of reoperations and/or recurrence of herniation. The mechanism of cord herniation may have been responsible for his delayed presentation approximately 5 months after his bilateral lung transplant operation.

\section{References}

1. Khattar NK, Donovan AM, Oxford BG, Adams SWC, Altstadt TJ. Traumatic ventral cervical spinal cord herniation: a case report. Cureus. 2019;11(2):e4070.

2. Francis $D$, Batchelor $P$, Gates P. Posttraumatic spinal cord herniation. J Clin Neurosci. 2006;13(5):582-586.

3. Papavero L, Engler N, Kothe R. Incidental durotomy in spine surgery: first aid in ten steps. Eur Spine J. 2015;24(9):2077-2084. 
4. Richter B, Xu C, Jho DH. Technical note: inlay-onlay sandwich graft technique for repairing durotomy in spine surgery for fragile dura. J Spine Neurosurg. 2016;5:3.

5. Zakaria R, Ellenbogen JR, Grewal IS, Buxton N. Posterior spinal cord herniation: a novel occurrence following surgery for an intramedullary cyst at the thoracolumbar junction. Eur Spine J. 2013; 22(suppl 3):S399-S403.

6. Rubin MN, Rabinstein AA. Vascular diseases of the spinal cord. Neurol Clin. 2013;31(1):153-181.

7. Krings T, Geibprasert S. Spinal dural arteriovenous fistulas. AJNR Am J Neuroradiol. 2009;30(4):639-648.

8. Le TC, Grunch BH, Karikari IO, et al. Dorsal thoracic spinal cord herniation: report of an unusual case and review of the literature. Spine J. 2012;12(10):e9-e12.

9. Nakashima $\mathrm{H}$, Ishikawa $\mathrm{Y}$, Kato $\mathrm{F}$, et al. Postoperative iatrogenic spinal cord herniation: three case reports with a literature review. Nagoya J Med Sci. 2020;82(2):383-389.

10. Nejat F, Cigarchi SZ, Kazmi SS. Posterior spinal cord herniation into an extradural thoracic arachnoid cyst: surgical treatment. Case report and review of the literature. J Neurosurg. 2006;104(3 suppl):210-211.

11. Diabira S, Henaux PL, Riffaud L, Hamlat A, Brassier G, Morandi X. Brown-Sequard syndrome revealing intradural thoracic disc herniation. Eur Spine J. 2011;20(1):65-70.

12. Koçak A, Kiliç A, Nurlu G, et al. A new model for tethered cord syndrome: a biochemical, electrophysiological, and electron microscopic study. Pediatr Neurosurg. 1997;26(3):120-126.

13. Henry A, Tunkel R, Arbit E, Ku A, Lachmann E. Tethered thoracic cord resulting from spinal cord herniation. Arch Phys Med Rehabil. 1997;78(5):530-533.

14. Miyake S, Tamaki N, Nagashima T, Kurata H, Eguchi T, Kimura H. Idiopathic spinal cord herniation. Report of two cases and review of the literature. J Neurosurg. 1998;88(2):331-335.

15. Filippidis AS, Kalani MY, Theodore N, Rekate HL. Spinal cord traction, vascular compromise, hypoxia, and metabolic derangements in the pathophysiology of tethered cord syndrome. Neurosurg Focus. 2010;29(1):E9.

16. Shin JH, Krishnaney AA. Idiopathic ventral spinal cord herniation: a rare presentation of tethered cord. Neurosurg Focus. 2010; 29(1):E10.

17. Yamada S, Zinke DE, Sanders D. Pathophysiology of "tethered cord syndrome." J Neurosurg. 1981;54(4):494-503.

18. Nakamura M, Fujiyoshi K, Tsuji O, et al. Long-term surgical outcomes of idiopathic spinal cord herniation. J Orthop Sci. 2011;16(4):347-351.

19. Carter BJ, Griffith BD, Schultz LR, Abdulhak MM, Newman DS, Jain R. Idiopathic spinal cord herniation: an imaging diagnosis with a significant delay. Spine J. 2015;15(9):1943-1948.

20. Eltoukhy M, Gkolemis C. Late-onset post-traumatic spinal cord herniation as a rare and overlooked cause of late neurologic deterioration after penetrating injury to the thoracic spine: a case report and review of the literature. World Neurosurg. 2020;142:408-412.

21. Watanabe $M$, Chiba K, Matsumoto M, Maruiwa H, Fujimura $Y$, Toyama Y. Surgical management of idiopathic spinal cord herniation: a review of nine cases treated by the enlargement of the dural defect. J Neurosurg. 2001;95(2 suppl):169-172.
22. Zairi F, Thines L, Bourgeois P, Dereeper O, Assaker R. Spinal cord herniation: a misdiagnosed and treatable cause of thoracic myelopathy. Acta Neurochir (Wien). 2010;152(11):1991-1996.

23. Kaliya-Perumal AK, Tan M, Nolan CP, Oh JYL. Post-surgical thoracic spinal cord herniation following an unrepaired dural defect: a rare complication. Br J Neurosurg. Published online September 10 2019. doi:https://doi.org/10.1080/02688697.2019.1661966

24. Moriyama T, Tachibana T, Maruo K, Inoue S, Okada F, Yoshiya S. Postoperative spinal cord herniation with pseudomeningocele in the cervical spine: a case report. Spine J. 2013;13(10):e43-e45.

25. Hosono N, Yonenobu K, Ono K. Postoperative cervical pseudomeningocele with herniation of the spinal cord. Spine (Phila Pa 1976). 1995;20(19):2147-2150.

26. Lee GYF, Paradiso G, Tator CH, Gentili F, Massicotte EM, Fehlings MG. Surgical management of tethered cord syndrome in adults: indications, techniques, and long-term outcomes in 60 patients. J Neurosurg Spine. 2006;4(2):123-131.

27. Heller RS, Hwang SW, Riesenburger RI. Dorsal cervical spinal cord herniation precipitated by kyphosis deformity correction for spinal cord tethering. World Neurosurg. 2017;100:709.e1-709.e4.

28. Watters MR, Stears JC, Osborn AG, et al. Transdural spinal cord herniation: imaging and clinical spectra. AJNR Am J Neuroradiol. 1998;19(7):1337-1344.

29. Kwon H, Lee S, Lee SH, Kim ES. Dural rupture and subsequent spinal cord herniation due to closed wound suction drainage after spinal surgery. Br J Neurosurg. 2021;12:1-3.

30. Abd Elwahab SM, O'Sullivan MJ. Spinal cord herniation after resection of cervical spinal neurofibroma with a unique presentation. Spine J. 2015;15(4):e1-e3.

31. Belen D, Er U, Gurses L, Yigitkanli K. Delayed pseudomyelomeningocele: a rare complication after foramen magnum decompression for Chiari malformation. Surg Neurol. 2009;71(3):357-361.

32. lencean SM, Poeata I. Late postoperative cervical spinal cord herniation. Spine J. 2014;14(5):856-857.

\section{Disclosures}

The authors report no conflict of interest concerning the materials or methods used in this study or the findings specified in this paper.

\section{Author Contributions}

Conception and design: all authors. Acquisition of data: Ogbu, Eltoukhy. Drafting the article: Ogbu. Critically revising the article: all authors. Reviewed submitted version of manuscript: all authors. Approved the final version of the manuscript on behalf of all authors: Ogbu. Administrative/technical/material support: Tzerakis. Study supervision: Tzerakis.

\section{Correspondence}

Ikenna Ogbu: Royal Stoke University Hospital, University Hospitals of North Midlands (UHNM), Staffordshire, West Midlands, United Kingdom. ikenna.ogbu@uhnm.nhs.uk. 